\title{
Top quark pair production and decay into 6 fermions at linear colliders
}

\author{
Karol Kołodziej \\ Institute of Physics, University of Silesia \\ ul. Uniwersytecka 4, PL-40007 Katowice, Poland
}

\begin{abstract}
The production of a $t \bar{t}$-pair and its decay into a 6 fermion final state of different flavours in $e^{+} e^{-}$ annihilation at centre of mass energies typical for linear colliders is analyzed in the framework of the Standard Model. The results of calculation based on exact matrix elements at the tree level and full 6 particle phase space are compared with a few different approximations. It is shown that the effects related to off-shellness of the $t \bar{t}$-pair and background contributions are sizable both in the continuum and at the threshold.
\end{abstract}

\footnotetext{
${ }^{1}$ Work supported in part by the Polish State Committee for Scientific Research (KBN) under contract No. 2 P03B 00418 and by European Commission's 5-th Framework contract HPRN-CT-2000-00149.

${ }^{2}$ E-mail: kolodzie@us.edu.pl
} 


\section{Introduction}

The physical properties of the top quark directly measured at the Tevatron are in a very good agreement with those derived from the Standard Model (SM) analysis of the data collected at LEP and SLC [1]. However, as the top quark is the heaviest particle ever observed, with the mass close to the scale of electroweak symmetry breaking, the measurement of its Yukawa coupling may give hints towards better understanding of the electroweak symmetry breaking mechanism and observed fermion mass hierarchy. Should the effects of the physics beyond the SM be visible at the energy scale below $1 \mathrm{TeV}$, it is very likely that precise measurements of the top couplings to electroweak gauge bosons, or its electric and magnetic dipole moment show deviations from the corresponding SM values. The high-precision of measurements of the top quark properties and interactions can be by far the best reached at an $e^{+} e^{-}$collider which operates at a clean experimental environment. Therefore, such measurements are planned at TESLA [2] and will most certainly belong to the research program of any future $e^{+} e^{-}$collider [3].

It is clear, that in order to disentangle the possible new physical effects from physics of the $\mathrm{SM}$, it is crucial to know the SM predictions for the top quark pair production and decay as precisely as possible. Due to the large mass and width, the top quark decays before toponium resonances can form and the predictions for

$$
e^{+} e^{-} \rightarrow t \bar{t}
$$

can be obtained within the perturbative QCD. The predictions for reaction (11) in the threshold region were obtained in [4] and then improved by calculation of the next-to-next-to-leading order QCD corrections [5], and by including the effects of initial state radiation and beamstrahlung [6]. The $\mathcal{O}\left(\alpha \alpha_{s}\right)$ [7] and $\mathcal{O}\left(\alpha \alpha_{s}^{2}\right)$ [8] corrections to the top decay into a $W$ boson and a $b$ quark are also known. In the continuum above the threshold, the QCD predictions for reaction (11) are known to order $\alpha_{s}^{2}$ [9] and the electroweak (EW) corrections to one-loop order [10], including the hard bremsstrahlung corrections [11. The QCD and EW corrections are large, typically of $\mathcal{O}(10 \%)$. Order $\alpha_{s} 13$ and $\alpha_{s}^{2} \mathrm{QCD}$, and EW corrections has been combined in [14].

As measurements of the top quark physical characteristics, in particular its static properties such as magnetic and electric dipole moments, will be performed at high energies, much above the $t \bar{t}$ threshold, it is crucial to know off-resonance background contributions to any specific 6 fermion decay channel and to estimate the effects related to the off-shellness of the $t \bar{t}$-pair. Therefore, in the present note, instead of considering production of the top quark pair (1) and its subsequent decay into a specific 6 fermion final state, the 6 fermion reactions of the form

$$
e^{+} e^{-} \rightarrow b f_{1} \bar{f}_{1}^{\prime} \bar{b} f_{2} \bar{f}_{2}^{\prime}
$$

where $f_{1}=\nu_{\mu}, \nu_{\tau}, u, c, f_{2}=\mu^{-}, \tau^{-}, d, s$, and $f_{1}^{\prime}, f_{2}^{\prime}$ are the corresponding weak isospin partners of $f_{1}, f_{2}, f_{1}^{\prime}=\mu^{-}, \tau^{-}, d, s, f_{2}^{\prime}=\nu_{\mu}, \nu_{\tau}, u, c$, are studied in the lowest order of SM. For the sake of simplicity, it is assumed that the actual values of $f_{1}$ and $f_{2}^{\prime}$ are different from each other,

and that neither $f_{1}^{\prime}$ nor $f_{2}$ is an electron. The results for reaction (2) are compared with the results obtained in a few different approximations: the double resonance approximation for $W$ bosons

$$
e^{+} e^{-} \rightarrow b W^{+*} \bar{b} W^{-*} \rightarrow b f_{1} \bar{f}_{1}^{\prime} \bar{b} f_{2} \bar{f}_{2}^{\prime}
$$


where only those 61 Feynman diagrams are taken into account which contribute to $e^{+} e^{-} \rightarrow$ $b W^{+} \bar{b} W^{-}$and the $\mathrm{W}$ bosons are considered as being off-mass-shell, the double resonance approximation for a $t$ - and $\bar{t}$-quark

$$
e^{+} e^{-} \rightarrow t^{*} \bar{t}^{*} \rightarrow b f_{1} \bar{f}_{1}^{\prime} \bar{b} f_{2} \bar{f}_{2}^{\prime}
$$

with only two 'signal' diagrams contributing and, finally, with 3 different narrow width approximations: for the $W$ bosons, top and antitop quarks, and a single top quark [15].

A similar analysis of the 6 fermion processes relevant for a $t \bar{t}$ production in $e^{+} e^{-}$annihilation have been performed in refs. [16], [17], where semileptonic channels of reaction (2) have been studied, and in ref. [18], where purely hadronic channels of (2) have been analysed. Moreover, irreducible QCD background to top searches in semileptonic channels of (2) has been discussed in [19]. The novelty of the present work, besides the more detailed discussion of the different approximations listed above, consists in taking into account both the electroweak and QCD lowest order contributions. Moreover, as light fermion masses are not neglected, the cross sections are calculated without any kinematical cuts.

Basics of the calculation are described in the next section. Numerical results are presented and discussed in Section 3 and, finally, in Section 4, the concluding remarks are given.

\section{Calculation}

The calculation of matrix elements of reaction (2) is based on the complete set of the Feynman diagrams at the tree level of SM. The number of diagrams which contribute to (2) in the unitary gauge, neglecting the Higgs boson coupling to fermions lighter than a $b$ quark, amounts to 201 for semi leptonic final states, which contain two different charged leptons, and to 333 for purely hadronic final states, with different quark flavours. The necessary matrix elements are calculated with the method proposed in [20] and further developed in [21]. As in [21], fermion masses are kept nonzero in the matrix elements and in the kinematics. The constant widths of unstable particles, the massive electroweak vector bosons, the Higgs boson and the top quark, are introduced through the complex mass parameters:

$$
M_{V}^{2}=m_{V}^{2}-i m_{V} \Gamma_{V}, \quad V=W, Z, \quad M_{H}^{2}=m_{H}^{2}-i m_{H} \Gamma_{H}, \quad M_{t}=m_{t}-i \Gamma_{t} / 2,
$$

which replace masses in the corresponding propagators, both in the $s$ - and $t$-channel Feynman diagrams,

$$
\Delta_{F}^{\mu \nu}(q)=\frac{-g^{\mu \nu}+q^{\mu} q^{\nu} / M_{V}^{2}}{q^{2}-M_{V}^{2}}, \quad \Delta_{F}(q)=\frac{1}{q^{2}-M_{H}^{2}}, \quad S_{F}(q)=\frac{\not q+M_{t}}{q^{2}-M_{t}^{2}} .
$$

Propagators of a photon and a gluon are taken in the Feynman gauge.

The 6 particle phase space of reaction (国)

$$
\mathrm{d}^{14} \text { Lips }=(2 \pi)^{4} \delta^{4}\left(p_{1}+p_{2}-\sum_{i=3}^{8} p_{i}\right) \prod_{i=3}^{8} \frac{\mathrm{d}^{3} p_{i}}{(2 \pi)^{3} 2 E_{i}},
$$

where the energies and momenta of the initial state particles of reaction (2) has been numbered from 1 to 2 , and those of the finale state particles from 3 to 8 , is parametrized in three different 
ways

$$
\begin{aligned}
\mathrm{d}^{14} \text { Lips } & =1 /(2 \pi)^{14} \mathrm{~d} P S_{2}\left(s, s_{345}, s_{678}\right) \mathrm{d} P S_{2}\left(s_{345}, m_{3}^{2}, s_{45}\right) \mathrm{d} P S_{2}\left(s_{678}, m_{6}^{2}, s_{78}\right) \\
& \times \mathrm{d} P S_{2}\left(s_{45}, m_{4}^{2}, m_{5}^{2}\right) \mathrm{d} P S_{2}\left(s_{78}, m_{7}^{2}, m_{8}^{2}\right) \mathrm{d} s_{345} \mathrm{~d} s_{678} \mathrm{~d} s_{45} \mathrm{~d} s_{78}, \\
\mathrm{~d}^{14} \text { Lips } & =1 /(2 \pi)^{14} \mathrm{~d} P S_{2}\left(s, s_{34}, s_{5678}\right) \mathrm{d} P S_{2}\left(s_{5678}, s_{56}, s_{78}\right) \mathrm{d} P S_{2}\left(s_{34}, m_{3}^{2}, m_{4}^{2}\right) \\
& \times \mathrm{d} P S_{2}\left(s_{56}, m_{5}^{2}, m_{6}^{2}\right) \mathrm{d} P S_{2}\left(s_{78}, m_{7}^{2}, m_{8}^{2}\right) \mathrm{d} s_{34} \mathrm{~d} s_{5678} \mathrm{~d} s_{56} \mathrm{~d} s_{78} .
\end{aligned}
$$

and

$$
\begin{aligned}
\mathrm{d}^{14} \text { Lips } & =1 /(2 \pi)^{14} \mathrm{~d} P S_{2}\left(s, m_{3}^{2}, s_{45678}\right) \mathrm{d} P S_{2}\left(s_{45678}, s_{45}, s_{678}\right) \mathrm{d} P S_{2}\left(s_{678}, m_{6}^{2}, s_{78}\right) \\
& \times \mathrm{d} P S_{2}\left(s_{45}, m_{4}^{2}, m_{5}^{2}\right) \mathrm{d} P S_{2}\left(s_{78}, m_{7}^{2}, m_{8}^{2}\right) \mathrm{d} s_{45678} \mathrm{~d} s_{45} \mathrm{~d} s_{678} \mathrm{~d} s_{78} .
\end{aligned}
$$

In Eqs. (810), $s_{i j k \ldots}=\left(p_{i}+p_{j}+p_{k}+\ldots\right)^{2}, i, j, k=3, \ldots, 8$, and $\mathrm{d} P S_{2}\left(s, s^{\prime}, s^{\prime \prime}\right)$ is a two particle (subsystem) phase space element defined by

$$
\mathrm{d} P S_{2}\left(s, s^{\prime}, s^{\prime \prime}\right)=\delta^{4}\left(p-p^{\prime}-p^{\prime \prime}\right) \frac{\mathrm{d}^{3} p^{\prime}}{2 E^{\prime}} \frac{\mathrm{d}^{3} p^{\prime \prime}}{2 E^{\prime \prime}}=\frac{\left|\vec{p}^{\prime}\right|}{4 \sqrt{s}} \mathrm{~d} \Omega^{\prime},
$$

where $\vec{p}^{\prime}$ is the momentum and $\Omega^{\prime}$ is the solid angle of one of the particles (subsystems) in the relative centre of mass system, $\vec{p}^{\prime}+\vec{p}^{\prime \prime}=0$. Using the rotational symmetry with respect to the beam line, an integration over one azimuthal angle in the c.m.s. becomes trivial. This reduces the number of necessary integrations to be performed.

Parametrization (8) is most suitable for integrating the dominant $t \bar{t}$ resonance contributions of Eq. (4). Parametrization (9) covers best contributions corresponding to the double $W$ resonance approximation of Eq. (3), whereas parametrization (10) covers other 'background' contributions to reaction (21). Parametrizations (8-10) are used with different permutations of external particle momenta and with different mappings which take into account the BreitWigner shape of the $W, Z$, Higgs and top quark resonances as well as the exchange of a massless photon or gluon. For a given final state and c.m.s. energy, altogether about 60 kinematical channels are sampled in order to find the dominant channels which contribute more than $0.1 \%$ to the total cross section. Those dominant kinematical channels are then used in a multichannel Monte Carlo (MC) integration routine.

The phase space integration is simplified in the narrow width approximations. The cross section of reaction (3) in the narrow $W$ width approximation is given by

$$
\sigma_{b W^{+} \bar{b} W^{-}}=\sigma\left(e^{+} e^{-} \rightarrow b W^{+} \bar{b} W^{-}\right) \Gamma\left(W^{+} \rightarrow f_{1} \bar{f}_{1}^{\prime}\right) \Gamma\left(W^{-} \rightarrow f_{2} \bar{f}_{2}^{\prime}\right) / \Gamma_{W}^{2} .
$$

Similarly, the cross section of reaction (4) in the narrow width approximation for the top and antitop reads

$$
\sigma_{t \bar{t}}=\sigma\left(e^{+} e^{-} \rightarrow t \bar{t}\right) \Gamma\left(t \rightarrow b f_{1} \bar{f}_{1}^{\prime}\right) \Gamma\left(\bar{t} \rightarrow \bar{b} f_{2} \bar{f}_{2}^{\prime}\right) / \Gamma_{t}^{2}
$$

Finally, in the approximation where only the top quark is put on its mass shell, the cross section is given by

$$
\sigma_{t \bar{b} f_{2} \bar{f}_{2}^{\prime}}=\sigma\left(e^{+} e^{-} \rightarrow t \bar{b} f_{2} \bar{f}_{2}^{\prime}\right) \Gamma\left(t \rightarrow b f_{1} \bar{f}_{1}^{\prime}\right) / \Gamma_{t} .
$$

There are 7 integrations which have to be performed numerically in order to obtain total cross sections in approximations (12) and (14) and only one integration in the case of approximation (13). All numerical integrations in the present work are performed with VEGAS [22]. 


\section{$3 \quad$ Numerical results}

In this section, numerical results for the total and a few differential cross sections of reaction (2) are presented. They are compared with the corresponding results obtained within approximations (3), (田) and (12 14).

The SM electroweak physical parameters are defined in terms of the gauge boson masses and widths, the top mass and the Fermi coupling constant. The actual values of the parameters are taken from [1]:

$$
\begin{gathered}
m_{W}=80.419 \mathrm{GeV}, \quad \Gamma_{W}=2.12 \mathrm{GeV}, \quad m_{Z}=91.1882 \mathrm{GeV}, \quad \Gamma_{Z}=2.4952 \mathrm{GeV}, \\
m_{t}=174.3 \mathrm{GeV}, \quad G_{\mu}=1.16639 \times 10^{-5} \mathrm{GeV}^{-2}
\end{gathered}
$$

The Higgs boson mass is assumed to be $m_{H}=115 \mathrm{GeV}$ and the Higgs width is calculated according to the lowest order of SM resulting in $\Gamma_{H}=4.9657 \mathrm{MeV}$. The top quark width is taken to be $\Gamma_{t}=1.5 \mathrm{GeV}$.

The SM electroweak coupling constants are given in terms of the electric charge $e_{W}=$ $\left(4 \pi \alpha_{W}\right)^{1 / 2}$ and electroweak mixing parameter $\sin ^{2} \theta_{W}$ with

$$
\alpha_{W}=\sqrt{2} G_{\mu} m_{W}^{2} \sin ^{2} \theta_{W} / \pi, \quad \sin ^{2} \theta_{W}=1-m_{W}^{2} / m_{Z}^{2},
$$

where $m_{W}$ and $m_{Z}$ are physical masses of the $W^{ \pm}$and $Z^{0}$ boson specified in Eq. (15). This kind of parametrization, together with substitutions of Eq. (5), is usually referred to as the 'fixed width scheme' (FWS). The strong coupling constant is given by $g_{s}=\left(4 \pi \alpha_{s}\left(M_{Z}\right)\right)^{1 / 2}$, with $\alpha_{s}\left(M_{Z}\right)=0.1185$.

It is also possible to perform computations with the complex electroweak mixing parameter

$$
\sin ^{2} \theta_{W}=1-M_{W}^{2} / M_{Z}^{2}
$$

with $M_{W}^{2}$ and $M_{Z}^{2}$ defined in Eq. (5). This kind of parametrization is called the 'complexmass scheme' (CMS) 23]. CMS has the advantage that it preserves the $S U(2) \times U(1)$ Ward identities [23].

For the sake of definiteness, other fermion masses used in the calculation are listed below [1]:

$$
\begin{gathered}
m_{e}=0.510998902 \mathrm{MeV}, \quad m_{\mu}=105.658357 \mathrm{MeV}, \quad m_{\tau}=1777.03 \mathrm{MeV} \\
m_{u}=5 \mathrm{MeV}, \quad m_{d}=9 \mathrm{MeV}, \quad m_{s}=150 \mathrm{MeV}, \quad m_{c}=1.3 \mathrm{GeV}, \quad m_{b}=4.4 \mathrm{GeV} .
\end{gathered}
$$

The Cabibo-Kobayashi-Maskawa mixing is neglected.

Matrix elements of $e^{+} e^{-} \rightarrow b W^{+} \bar{b} W^{-}$and $e^{+} e^{-} \rightarrow t \bar{t}$ have been checked against MADGRAPH 25] showing an agreement up to 13-16 decimals. As the version of MADGRAPH used in the comparisons is not applicable to processes with 6 particles in the final state, it has been not possible to compare directly matrix elements of reactions (2) with those generated by MADGRAPH. Instead of that, matrix elements of different 'subprocesses' of (国), namely $e^{+} e^{-} \rightarrow$ $b f_{1} \bar{f}_{1}^{\prime} \bar{b} W^{-}, e^{+} e^{-} \rightarrow b W^{+} \bar{b} f_{2} \bar{f}_{2}^{\prime}$ and $e^{+} e^{-} \rightarrow f_{1} \bar{f}_{1}^{\prime} f_{2} \bar{f}_{2}^{\prime} Z$, have been compared successfully. The multichannel phase space generation routine has been checked by comparing normalization of 
different channels against each other and testing energy-momentum conservation and on-massshell relations. For several total cross sections, the numerical integration has been performed with different parametrizations of the phase space and the results have been stable within one standard deviation.

The standard deviation of the multichannel integration routine is obtained as a sum of the standard deviations calculated by VEGAS for individual channels. This gives a more conservative estimate of the integration error than for example adding up partial errors in quadrature.

Another test is a comparison with existing calculations. Results for total cross sections of $e^{+} e^{-} \rightarrow b \nu_{\mu} \mu^{+} \bar{b} d \bar{u}$ in the lowest order SM are compared with the results of [17] in Table 1. As in [17, , only the pure electroweak diagrams and the two $t \bar{t}$ signal diagrams are taken into account in $\sigma_{\text {all EW }}$ and $\sigma_{t^{*} t^{*}}$, respectively. For the sake of comparison, the physical parameters of [17] have been used, i.e. $m_{Z}=91.187 \mathrm{GeV}, \Gamma_{Z}=2.49 \mathrm{GeV}, m_{W}=80.22 \mathrm{GeV}, \Gamma_{Z}=2.052 \mathrm{GeV}$, $m_{t}=174 \mathrm{GeV}, \Gamma_{t}=1.558 \mathrm{GeV}, m_{b}=4.1 \mathrm{GeV}, m_{u}=2 \mathrm{MeV}$ and $m_{d}=5 \mathrm{MeV}$. The electroweak mixing parameter is defined as in Eq. (16) and $\alpha_{W}=1 / 128.07$ is used at the same time. As the values of the Higgs boson mass and width used in the calculation are not quoted in [17], $m_{H}=115 \mathrm{GeV}$ and the lowest order $\mathrm{SM}$ value $\Gamma_{H}=4.3977 \mathrm{MeV}$, corresponding to parameters of [17], have been used in Table 1. Another source of ambiguity in the comparison is the treatment of the finite widths of unstable particles which is not explicitly described in [17. Therefore the prescription of Eqs. (5) and (6) have been adopted. The results for $\sigma_{\text {all EW }}$ and $\sigma_{t^{*} \bar{t}^{*}}$ are shown in columns 2 and 3, whereas the corresponding results of the present work are shown in columns 4 and 5 . The results for $\sigma_{t^{*} \bar{t}^{*}}$ agree nicely within the uncertainties quoted in parenthesis. The agreement is still nice for the complete electroweak cross sections $\sigma_{\text {all EW }}$ above the $t \bar{t}$ threshold. Below the threshold, at $\sqrt{s}=340 \mathrm{GeV}$, there is a substantial

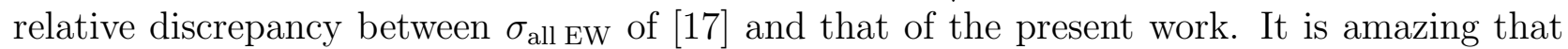
the results for all the approximated cross sections listed in Table 2 of [17] agree with the present work, also at $\sqrt{s}=340 \mathrm{GeV}$. It is difficult to state definitely what the actual reason for this discrepancy is. However, most probably it is the Higgs boson contribution, and in particular the Higgs-strahlung 'subrocess' $e^{+} e^{-} \rightarrow Z H$ with the Higgs boson decaying into a virtual $W^{+} W^{-}$-pair that is responsible for it. The results of the present work without the Higgs contribution are shown in the last column of Table 1. They nicely agree with the results of [17] which contain the Higgs with its mass and width not being specified.

Unfortunately, a similar detailed comparison with results of 18 is not possible, as the authors do not specify numerical values of the physical parameters used in their computations. As the cross section of reaction (2) at tree level is of $\mathcal{O}\left(\alpha_{W}^{6}\right)$, it is very sensitive to the choice of initial parameters. Although it is meaningless to perform any quantitative comparison, the results of the present work are in a qualitative agreement with those of ref. 18 which will be shown later. A detailed quantitative comparison with Accomando, Ballestrero and Pizzio [16] is also not possible as the authors include some radiative effects in most of their results. A meaningful comparison could in principle be performed for the Born cross sections of $e^{+} e^{-} \rightarrow b \nu_{\mu} \mu^{+} \bar{b} d \bar{u}$ corresponding to the $t \bar{t}$ signal and background at $\sqrt{s}=500 \mathrm{GeV}$. With cuts of [16] and the physical parameters of the present work, one obtains 17.895(9) fb and 1.25(2) fb for the signal and background, respectively. The result for the signal cross section differs from that of [16] by about $2 \%$ while the relative difference between the background cross sections is much bigger, probably because there is no gluon exchange contribution included in the Born background cross section of [16].

Lowest order SM total cross sections of the semileptonic channel $e^{+} e^{-} \rightarrow b \nu_{\mu} \mu^{+} \bar{b} d \bar{u}$ of reaction 
Table 1: Comparison of the lowest order SM total cross sections of $e^{+} e^{-} \rightarrow b \nu_{\mu} \mu^{+} \bar{b} d \bar{u}$ of [17 and present work. Results of [17] obtained with a complete set of the electroweak diagrams, $\sigma_{\text {all EW }}$, and two $t \bar{t}$ signal diagrams, $\sigma_{t^{*} \bar{t}^{*}}$, are shown in columns 2 and 3 , whereas the corresponding results of the present work are shown in columns 4 and 5. Here the parameters of [17] are used. All cross sections are in $\mathrm{fb}$. The number in parenthesis show the uncertainty of the last decimal.

\begin{tabular}{|c|c|c|c|c|c|}
\hline \multirow{2}{*}{$\sqrt{s}$} & F. Yuasa, et. al. [17 & \multicolumn{3}{|c|}{ Present Work } \\
\cline { 2 - 6 }$(\mathrm{GeV})$ & $\sigma_{\text {all EW }}$ & $\sigma_{t^{*} \bar{t}^{*}}$ & $\sigma_{\text {all EW }}$ & $\sigma_{t^{*} \bar{t}^{*}}$ & $\sigma_{\text {all EW }}^{\text {no Higgs }}$ \\
\hline 340 & $0.687(2)$ & $0.4462(3)$ & $0769(10)$ & $0.4455(4)$ & $0.689(2)$ \\
350 & $6.45(1)$ & $6.187(4)$ & $6.59(2)$ & $6.175(4)$ & $6.45(1)$ \\
360 & $14.97(2)$ & $14.63(1)$ & $15.03(5)$ & $14.623(9)$ & $14.97(3)$ \\
380 & $21.42(4)$ & $21.00(1)$ & $21.48(8)$ & $20.99(1)$ & $21.49(5)$ \\
500 & $22.32(4)$ & $21.30(1)$ & $22.55(4)$ & $21.27(1)$ & $22.32(5)$ \\
\hline
\end{tabular}

(2) at different c.m.s. energies typical for future linear colliders are shown in Table 2. The complete lowest order result $\sigma$, the approximation of Eq. (3) $\sigma_{b W^{+*} \bar{b} W^{-*}}$, the narrow $W$ width approximation of Eq. (12) $\sigma_{b W^{+} \bar{b} W^{-}}$, the approximation of Eq. (田) $\sigma_{t^{*} \bar{t}^{*}}$, the narrow width approximation of Eq. (13) for a top and an antitop quark $\sigma_{t \bar{t}}$ and the narrow width approximation for a top quark of Eq. (14) $\sigma_{t \bar{b} d \bar{u}}$ have been all obtained in FWS. The SM tree level analytic expression for the partial widths of the $W$ boson and the experimental value of total $W$ width $\Gamma_{W}$ have been used in Eq. (12). Similarly, the SM tree level analytic expression for the partial widths of the $t$ quark in the zero fermion mass approximation [24] and the total top width $\Gamma_{t}=1.5 \mathrm{GeV}$ have been used in Eqs. (13) and (14). The use of these values of $\Gamma_{W}$ and $\Gamma_{t}$ in Eqs. (12 14) is preferred in the comparison because the same values have been used in substitutions of Eq. (5). In Table 2, the numbers in parenthesis are standard deviations of the MC integration, which show an uncertainty of the last decimal.

A cross section of the 6 fermion reaction $e^{+} e^{-} \rightarrow b \nu_{\mu} \mu^{+} \bar{b} d \bar{u}$ is nonzero already below the $t \bar{t}$-pair production threshold. It is the single top (antitop) resonance and nonresonant background contributions which are responsible for that effect. Whether this background may affect physical observables in the threshold region, such as the top invariant mass distribution or angular distributions of the final state quarks or leptons, will be discussed later. Close to threshold, at $\sqrt{s}=360 \mathrm{GeV}$, the relative difference between $\sigma$ and the narrow width approximation $\sigma_{t \bar{t}}$ is about $-1.5 \%$, whereas in the continuum the difference becomes bigger, as relevant as radiative corrections, amounting to $7 \%$ at $\sqrt{s}=500 \mathrm{GeV}$ and $19 \%$ at $\sqrt{s}=800 \mathrm{GeV}$. At higher energies, the difference between $\sigma$ and $\sigma_{t \bar{t}}$ becomes so large that approximation (13) does not make sense any more. Comparison of approximated results $\sigma_{b W^{+*} \bar{b} W^{-*}}$ and $\sigma_{b W^{+} \bar{b} W^{-}}$ with the complete result $\sigma$ shows that approximations of Eqs. (3) and (12) are relatively much better in a wide range of the c.m.s. energy from $360 \mathrm{GeV}$ to $2 \mathrm{TeV}$.

The pure off-shellness effects of the $t \bar{t}$-pair can be regarded as the difference between approximations $\sigma_{t^{*} \bar{t}^{*}}$ of Eq. (田) and $\sigma_{t \bar{t}}$ of Eq. (13). They are plotted in Fig. 1 as a function of the c.m.s. energy. The two plots in Fig. 1 show a similar behaviour with c.m.s. energy as those in Fig. 4 of F. Gangemi et. al. [18. A naive multiplication of the results plotted in Fig. 1 and 
Table 2: Lowest order SM total cross sections of $e^{+} e^{-} \rightarrow b \nu_{\mu} \mu^{+} \bar{b} d \bar{u}$ in $\mathrm{fb}$ at different c.m.s. energies: the complete lowest order result $\sigma$, the approximation of Eq. (3) $\sigma_{b W^{+*} \bar{b} W^{-*}}$, the narrow $W$ width approximation of Eq. (12) $\sigma_{b W^{+} \bar{b} W^{-}}$, the approximation of Eq. (14) $\sigma_{t^{*} \bar{t}^{*}}$, the narrow width approximation of Eq. (13) for a top and an antitop quark $\sigma_{t \bar{t}}$ and the narrow width approximation for top quark of Eq. (14) $\sigma_{t \bar{b} d \bar{u}}$. The number in parenthesis show the uncertainty of the last decimal.

\begin{tabular}{|c|c|c|c|c|c|c|}
\hline$\sqrt{s}(\mathrm{GeV})$ & $\sigma$ & $\sigma_{b W^{+*} \bar{b} W^{-*}}$ & $\sigma_{b W^{+} \bar{b} W^{-}}$ & $\sigma_{t^{*} \bar{t}^{*}}$ & $\sigma_{t \bar{t}}$ & $\sigma_{t \bar{b} d \bar{u}}$ \\
\hline 340 & $1.162(7)$ & $0.681(6)$ & $0.671(1)$ & $0.3521(2)$ & - & $0.2546(3)$ \\
\hline 360 & $13.64(2)$ & $13.224(8)$ & $13.618(8)$ & $12.79(1)$ & 13.875 & $13.42(1)$ \\
\hline 500 & $20.48(9)$ & $20.17(1)$ & $20.79(1)$ & $19.06(1)$ & 19.223 & $19.51(3)$ \\
\hline 800 & $10.61(4)$ & $10.46(3)$ & $10.75(1)$ & $9.181(5)$ & 8.918 & $9.47(1)$ \\
\hline 1000 & $7.35(4)$ & $7.33(4)$ & $7.54(1)$ & $6.171(4)$ & 5.862 & $6.390(7)$ \\
\hline 2000 & $2.43(2)$ & $2.48(3)$ & $2.48(1)$ & $1.847(2)$ & 1.510 & $1.822(2)$ \\
\hline
\end{tabular}

the results for $\sigma_{t^{*} \bar{t}^{*}}$ of Table 2 by a factor 12, corresponding to the different colour factor and the sum over 4 different hadronic channels, gives nice agreement with the signal cross section plotted in Fig. 4 of [18.

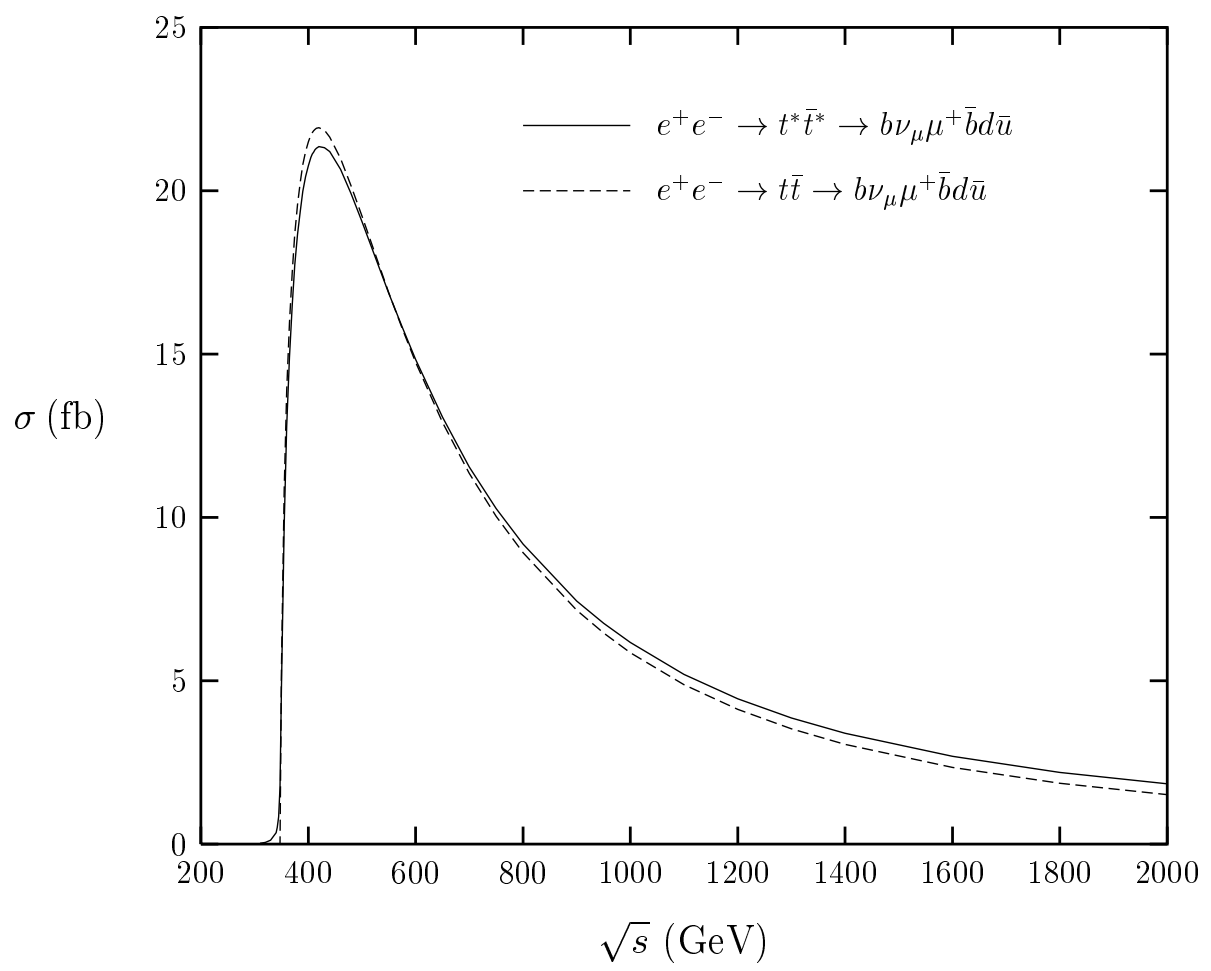

Figure 1. Total cross sections of $e^{+} e^{-} \rightarrow b \nu_{\mu} \mu^{+} \bar{b} d \bar{u}$ in approximations of Eqs. (4) (solid line) and (13) (dashed line) as functions of the c.m.s. energy.

Lowest order SM total cross sections for different channels of (2) at c.m.s. energies typical for TESLA are compared in Table 3. The cross sections of $e^{+} e^{-} \rightarrow b c \bar{s} \bar{b} d \bar{u}$ is about 3 times 
Table 3: Lowest order SM total cross sections in $\mathrm{fb}$ for different top production channels at c.m.s. energies typical for TESLA. The number in parenthesis show the uncertainty of the last decimal.

\begin{tabular}{|c|c|c|c|}
\hline$\sqrt{s}(\mathrm{GeV})$ & $e^{+} e^{-} \rightarrow b \nu_{\mu} \mu^{+} \bar{b} \tau^{-} \bar{\nu}_{\tau}$ & $e^{+} e^{-} \rightarrow b \nu_{\mu} \mu^{+} \bar{b} d \bar{u}$ & $e^{+} e^{-} \rightarrow b c \bar{s} \bar{b} d \bar{u}$ \\
\hline 360 & $4.36(1)$ & $13.65(4)$ & $42.1(2)$ \\
500 & $6.70(2)$ & $20.48(9)$ & $62.2(2)$ \\
800 & $3.43(2)$ & $10.61(4)$ & $32.1(1)$ \\
\hline
\end{tabular}

bigger than the cross section of $e^{+} e^{-} \rightarrow b \nu_{\mu} \mu^{+} \bar{b} d \bar{u}$, which in turn is about 3 times bigger than the cross section of $e^{+} e^{-} \rightarrow b \nu_{\mu} \mu^{+} \bar{b} \tau^{-} \bar{\nu}_{\tau}$. This reflects the relative numbers of colour degrees of freedom. Small deviations of the relative factors from 3 result from the gluon exchange contributions, which are absent for $e^{+} e^{-} \rightarrow b \nu_{\mu} \mu^{+} \bar{b} \tau^{-} \bar{\nu}_{\tau}$ and are different for $e^{+} e^{-} \rightarrow b \nu_{\mu} \mu^{+} \bar{b} d \bar{u}$ and $e^{+} e^{-} \rightarrow b c \bar{s} \bar{b} d \bar{u}$. The errors given in parenthesis have been obtained in the same way and have the same meaning as those of Tables 1 and 2 .

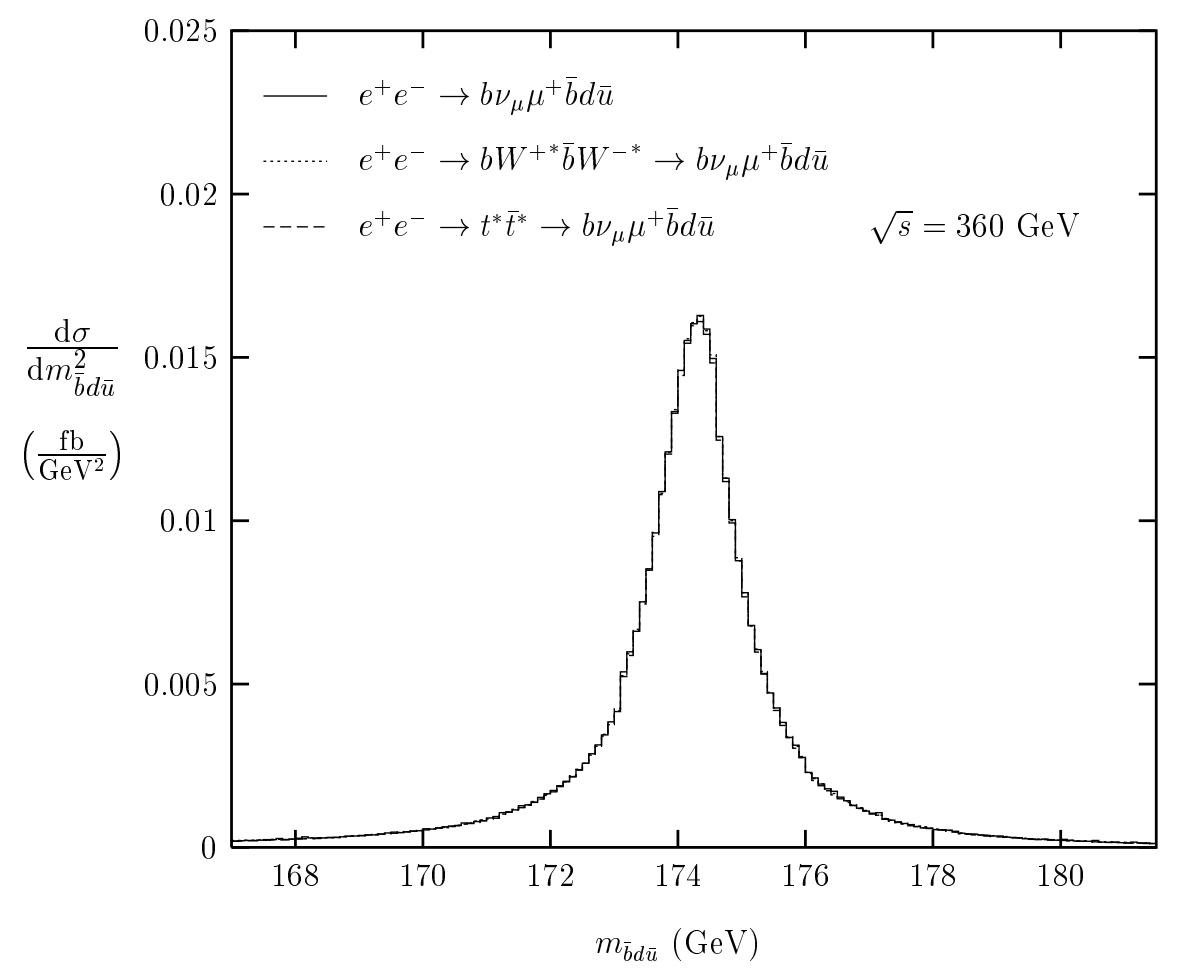

Figure 2. Differential cross sections $\mathrm{d} \sigma / \mathrm{d} m_{\bar{t}}^{2}$ of $e^{+} e^{-} \rightarrow b \nu_{\mu} \mu^{+} \bar{b} d \bar{u}$ at $\sqrt{s}=360 \mathrm{GeV}$ versus the invariant mass of the $\bar{t}$ quark reconstructed from the $\bar{b} d \bar{u}$ system (solid histogram) and from the $\bar{b} W^{-}$system in the narrow $W$ width approximation (dotted histogram).

How the background nonresonant contributions affect differential cross sections of (2) is illustrated in Figs. 2 and 3. In Fig. 2, the differential cross sections $\mathrm{d} \sigma / \mathrm{d} m_{\bar{b} d \bar{u}}^{2}$ of $e^{+} e^{-} \rightarrow b \nu_{\mu} \mu^{+} \bar{b} d \bar{u}$ at $\sqrt{s}=360 \mathrm{GeV}$ are plotted versus the invariant mass of the $\bar{t}$ quark reconstructed from the $\bar{b} d \bar{u}$ system. The three histograms: solid, corresponding to the complete lowest order result, 
dotted, corresponding to the approximation of Eq. (3), and dashed one, representing the $t \bar{t}$ signal (州), shown in Fig. 2, are almost indistinguishable. This means that approximation (4) is satisfactory and the background contributions coming from the single top (antitop) resonance and the nonresonant Feynman diagrams is negligible in this case.

The differential cross sections $\mathrm{d} \sigma / \mathrm{d} \cos \theta$ of $e^{+} e^{-} \rightarrow b \nu_{\mu} \mu^{+} \bar{b} d \bar{u}$ at $\sqrt{s}=360 \mathrm{GeV}$ are plotted versus cosine of the $\mu^{+}$(up going curves) and $d$ (down going curves) angle with respect to the positron beam in Fig. 3. The angular distributions obtained with the complete set of tree level Feynman diagrams differ substantially from the distributions based on approximations of Eqs. (3) and (4). The final state muon $\mu^{+}$(down quark $d$ ) goes more preferably in the direction of initial positron (electron) than it would follow from the approximated distributions based on Eqs. (3) and (4).

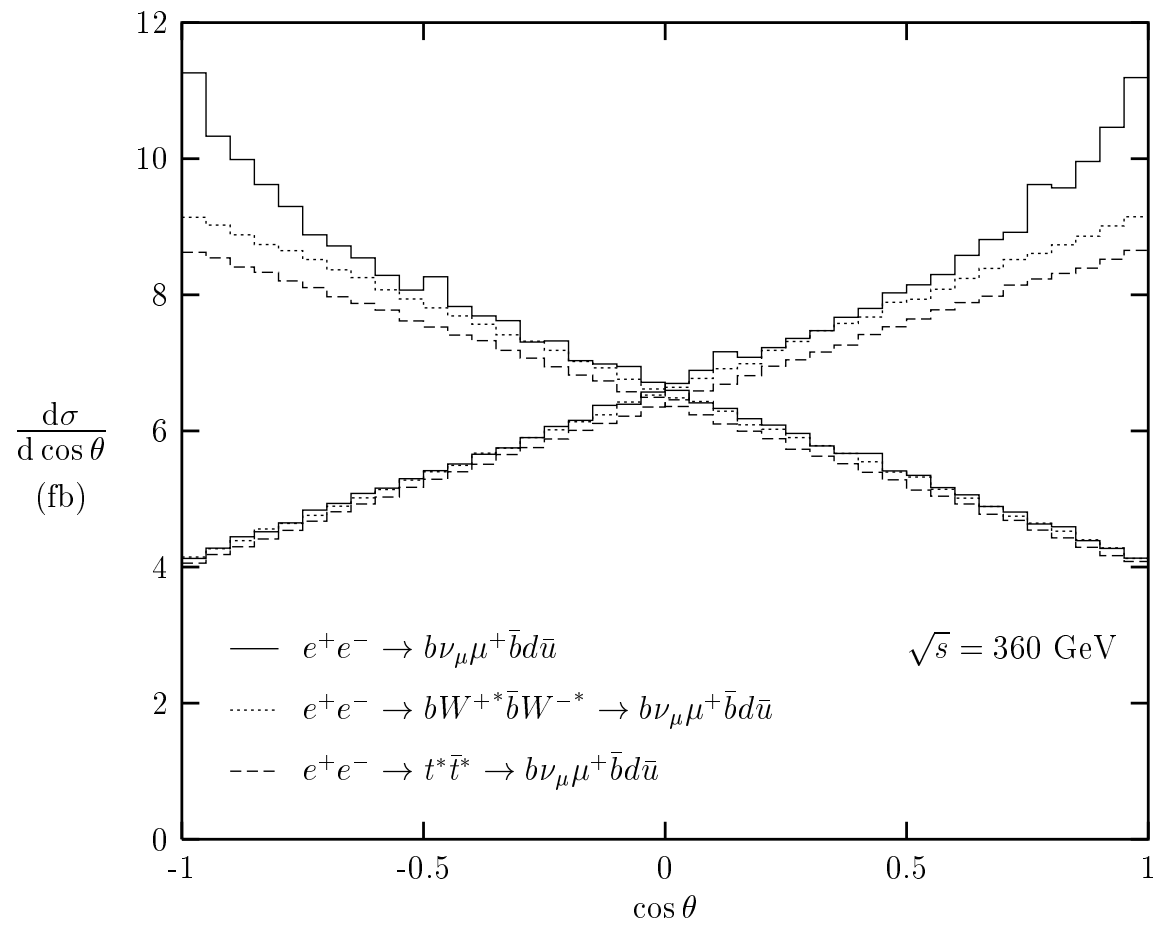

Figure 3. Differential cross sections of $e^{+} e^{-} \rightarrow b \nu_{\mu} \mu^{+} \bar{b} d \bar{u}$ at $\sqrt{s}=360 \mathrm{GeV}$ versus cosine of the $\mu^{+}$ (up going curve) and $d$ (down going curve) angle with respect to the positron beam.

\section{Summary and Outlook}

The production of a $t \bar{t}$-pair and its decay into a 6 fermion final state of different flavours in $e^{+} e^{-}$annihilation at energies typical for linear colliders has been analyzed in the framework of the SM. The results of calculation based on exact matrix elements at the tree level and full 6 particle phase space have been compared with the results obtained within a few different approximations: the double resonant approximations for the $W$ bosons (3) and for the top and antitop quarks (4), the narrow width approximation for the $W$ bosons (12), the narrow width approximation for the $t$ - and $\bar{t}$-quark (13) and for the $t$-quark quark only (14). 
It has been shown that the effects related to the off-shellness of the $t \bar{t}$-pair and to presence of background contributions to cross sections of six fermion reactions (2) are quite substantial. They are at the level of a few per cent already in the $t \bar{t}$ threshold region. In the continuum, at higher energies, the effects become quite sizable, reaching about $20 \%$ at $\sqrt{s}=800 \mathrm{GeV}$. Therefore, for achieving the desired precision level in the analysis of experimental data from linear colliders, it is mandatory to include them in theoretical predictions together with radiative corrections. The inclusion of the latter should reduce the dependence on the choice

of initial parameters mentioned in Section 3 in the context of comparisons with the existing calculations.

\section{References}

[1] Particle Data Group, D.E. Groom et al., Eur. Phys. J. C15 (2000) 1.

[2] TESLA Technical Design Report, Part III: Physics at an $e^{+} e^{-}$Linear Collider, edited by R.-D. Heuer, D. Miller, F. Richard, P.M. Zerwas, DESY 2001-011, ECFA 2001-209, TESLA Report 2001-23, TESLA-FEL 2001-05, March 2001, hep-ph/0106315; $e^{+} e^{-}$Collisions at TeV Energies: The Physics Potential, Proceedings of the Workshop, Annecy, Gran Sasso, Hamburg, February 1995 - September 1995, edited by P.M. Zerwas, DESY 96-123D, (1996) 1.

[3] T. Abe et al., American Linear Collider Working Group Collaboration, SLAC-R-570, Resource book for Snowmass 2001.

K. Abe et al., hep-ph/0109166.

[4] V.S. Fadin, V.A. Khoze, JETP Lett. 46, (1987) 525 and Sov. J. Nucl. Phys. 48 (1988) 309 ;

M.J. Strassler, M.E. Peskin, Phys. Rev. D43 (1991) 1500;

M. Jeżabek, J.H. Kühn, T. Teubner, Z. Phys. C56 (1992) 653;

Y. Sumino, K. Fuji, K. Hagiwara, M. Murayama, C.K. Ng, Phys. Rev. D47 (1992) 56.

[5] A.H. Hoang, T. Teubner, Phys. Rev. D58 (1998) 114023 and Phys. Rev. D60 (1999) 114027;

K. Melnikov, A. Yelkhovsky, Nucl. Phys. B528 (1998) 59;

O. Yakovlev, Phys. Lett. B457 (1999) 170; M. Beneke, A. Signer, V. Smirnov, Phys. Lett. B454 (1999) 137;

T. Nagano, A. Ota, Y. Sumino, Phys. Rev. D60 (1999) 114014;

A.H. Hoang, A.V. Manohar, I.W. Stewart, T. Teubner, Phys. Rev. Lett. 86 (2001) 1951 and hep-ph/0107144;

A.A. Penin, A.A. Pivovarov, Phys. Atom. Nucl. 64 (2001) 275;

A.H. Hoang et.al, Eur. Phys. J. direct C3 (2000) 1.

[6] D. Peralta, M. Martinez, R. Miquel, Top Mass Measurement at the $t \bar{t}$ Threshold, in. Proc. of the International Workshop on Linear Colliders (LCWS99) Sitges, May 1999.

[7] M. Jeżabek, J.H. Kühn, Nucl. Phys. B314 (1989) 1; A. Denner, T. Sack, Nucl. Phys. B358 (1991) 46; G. Eilam, R.R. Mendel, R. Migneron, A. Soni, Phys. Rev. Lett. 66 (1991) 3105. 
[8] A. Czarnecki and K. Melnikov, Nucl. Phys. B544 (1999) 520.

K.G. Chetyrkin, R. Harlander, T. Seidensticker, M. Steinhauser, Phys. Rev. D60 (1999) 114015 .

[9] K.G. Chetyrkin, J.H. Kühn, M. Steinhauser, Nucl. Phys. B482 (1996) 213;

R. Harlander, M. Steinhauser, Eur. Phys. J. C2 (1998) 151.

[10] B.Grzadkowski et al. , Nucl.Phys. B281 (1987) 18;

W. Beenakker, S.C. van der Marck, W. Hollik, Nucl Phys. B365 (1991) 24;

R.J. Guth and J.H. Kühn, Nucl.Phys. B368 (1992) 38;

W. Beenakker, A. Denner, A. Kraft, Nucl. Phys. B410 (1993) 219. V. Driesen, W. Hollik, A. Kraft, hep-ph/9603398, in $e^{+} e^{-}$Collisions at TeV Energies: The Physics Potential, Proceedings of the Workshop, Annecy, Gran Sasso, Hamburg, February 1995 - September 1995, edited by P.M. Zerwas, DESY 96-123D, (1996) 33.

[11] A.A. Akhundov, D.Yu. Bardin, A. Leike, Phys. Lett. B261 (1991) 321.

[12] R.J. Guth, J.H. Kühn, Nucl. Phys. B368 (1992) 38.

[13] J. Jersak, E. Laerman, P.M. Zerwas, Phys. Rev. D25 (1982) 1218; Erratum Phys. rev. D36 (1987) 310.

[14] J.H. Kühn, T. Hahn, R. Harlander, hep-ph/9912262.

[15] A. Biernacik, K. Ciećkiewicz, K. Kołodziej, Eur. Phys. J. C20 (2001) 233.

[16] E. Accomando, A. Ballestrero, M. Pizzio, Nucl.Phys. B512 (1998) 19.

[17] F. Yuasa, Y. Kurihara, S. Kawabata, Phys. Lett. B414 (1997) 178.

[18] F. Gangemi, G. Montagna, M. Moretti, O. Nicrosini, F. Piccinini, Nucl.Phys. B559 (1999) 3.

[19] S. Moretti, Eur. Phys. J. C9 (1999) 229.

[20] K. Kołodziej, M. Zrałek, Phys. Rev. D43 (1991) 3619.

[21] F. Jegerlehner, K. Kołodziej, Eur. Phys. J. C12 (2000) 77.

[22] G.P. Lepage, J. Comp. Phys. 27 (1978) 192.

[23] A. Denner, S. Dittmaier, M. Roth, D. Wackerroth, Nucl. Phys. B560 (1999) 33.

[24] E. Boos, M. Dubinin, M. Sachwitz, H.J. Schreiber, Eur. Phys. J. C16 (2000) 269.

[25] T. Stelzer, W.F. Long, Comput. Phys. Commun. 81 (1994) 357; E. Murayama, I. Watanabe, K. Hagiwara, KEK report 91-11, 1992. 